\title{
CHARACTERISTICS OF A LIQUID MICROLAYER FORMED BY A CONFINED VAPOR BUBBLE IN MICRO GAP BOILING BETWEEN TWO PARALLEL PLATES
}

\author{
Yaohua Zhang ${ }^{1}$ and Yoshio Utaka ${ }^{2 *}$ \\ 1. Technology Development Division Headquarters, NSK, Fujisawa, Japan \\ 2. Faculty of Engineering, Yokohama National University, Yokohama, Japan
}

\author{
*Author for correspondence \\ E-mail: utaka@ynu.ac.jp
}

\section{ABSTRACT}

The microlayer thickness formed between an elongated bubble and the heating surface during boiling in a parallel microchannel was measured directly using the laser extinction method. Microchannels with gap sizes of $s=0.15,0.30$, and 0.50 mm were used as test channels. Water, ethanol, toluene, and HFE7200 were used as testing fluids. The effects of gap sizes, the velocity of the bubble forefront, and the distance from the bubble inception site were investigated. Furthermore, the progress of bubble growth in two dimensions with acceleration was simulated by the volume of fluid (VOF) method using the computational fluid dynamics (CFD) package of FLUENT12.1. The microlayer thickness calculated from the simulation results shows relatively good agreement with the experimental results. The effects of the physical properties on the configuration of the microlayer were analyzed, and the characteristics of the microlayer in low- and high-velocity regions were qualitatively clarified. An empirical correlation for the microlayer thickness as the function of the Capillary number, the Weber number, and the Bond number in parallel mini/microchannels was proposed using dimension analysis. The present correlation predicts the experimental results within an accepted error range.

Keywords: Boiling, Microchannel, Microlayer thickness, Laser extinction method, Nondimensional correlation 


\section{INTRODUCTION}

Boiling in mini-/microscale channels is becoming increasingly important in various applications because this technique is capable of removing large amounts of heat over small areas. However, phase-change heat transfer mechanisms and characteristics at the microscale are distinctly different from those at the macroscale. In microchannels, bubbles nucleate and quickly grow to the channel size such that elongated bubbles that are confined by the channel walls are formed, and these bubbles grow very quickly in length, with a dynamic tip. A microlayer is formed between the bubbles and the inner heating plate walls after the dynamic tip of the bubble. The bubble inside the microchannel is confined such that the tip is an extremely small part, whereas most of the bubble is surrounded by a microlayer that is approximately parallel to the heating plate. The transient evaporation of the microlayer underneath the elongated bubbles is considered to be a major heat transfer mechanism. As such, the microlayer formed by the movement of the vapor-liquid interface has been investigated theoretically and experimentally in numerous studies.

Taylor [1] conducted experiments on a glass tube with a diameter of 2 to $3 \mathrm{~mm}$ filled with a glycerin-water solution that could be varied to cover a wide range of capillary numbers and measured the amount of liquid remaining on the tube wall after an air bubble propagated through the glass tube. The thickness of the film deposited on the wall by the propagating bubble was demonstrated to increase with an increase in capillary number. Bretherton [2] theoretically derived a prediction method for the microlayer thickness based on a lubrication approximation for the limit of $R e<<1$ while neglecting gravitational forces. Bretherton suggested that the liquid layer thickness could scale with the capillary number. For the limit of slow flow, Aussillous and Quere [3] proposed a firstorder analysis using scaling arguments. Based on their experimental results, a correlation of microlayer formation at the steady highvelocity region was proposed as $\delta / r=1.34 \mathrm{Ca}^{2 / 3} /\left(1+1.34 \times 2.5 \mathrm{Ca}^{2 / 3}\right)$, where $\delta$ is the thickness of the microlayer; $r$ is the radius of the microtube; $C a$ is the Capillary number $\left(=\mu_{L} V_{L} / \sigma\right)$, where $\sigma, \mu_{L}$, and $V_{L}$ are the surface tension, viscosity, and velocity of the bubble forefront, respectively, and coefficient 1.34 was derived by Bretherton [2]; and the coefficient 2.5 is empirical. The mechanism of microlayer formation under steady, adiabatic conditions has nearly been clarified. Under a slow-motion limit of bubbles with negligible inertia, the microlayer thickness is determined by the balance between the viscous and capillary forces near the bubble tip; thus, the microlayer thickness (normalized by the tube radius or gap size) is only dependent on the capillary number $C a$ and approaches a finite value as $\mathrm{Ca}$ increases. As the steady propagation speed of a bubble increases, the inertial effects cannot be neglected in the problem. The inertial term has to be taken into account, and we must employ the steady Navier-Stokes equation instead of the Stokes equation to describe the problem [4]. Aussillous and Quere [3] also investigated the inertia effect using lowviscosity liquids and found that above a threshold of $\mathrm{Ca}$, the microlayer is thicker than the correlation derived from the Stokes 
equation. They analyzed the problem by scaling the steady Navier-Stokes equation and found that the nondimensional microlayer thickness could be correlated as

$$
\frac{\delta}{r} \square \frac{C a^{2 / 3}}{1+C a^{2 / 3}-W e}
$$

which agrees with the trend that the inertia thickens the film thickness; however, it seems that the coefficient and exponent require modification based on the experimental or numerical results. Otherwise, this is only valid for $W e<1$.

Using a laser focus displacement meter, Han and Shikazono [5] measured the thickness of a microlayer formed in adiabatic slug flow in microtubes with diameters of $0.3,0.5,0.7,1.0$, and $1.3 \mathrm{~mm}$. They confirmed that the liquid microlayer thickness is determined only by the capillary number at a small $\mathrm{Ca}$ and the effect of the inertial force on the microlayer thickness is negligible. However, as $\mathrm{Ca}$ increases, the effect of inertia is not neglected. Based on the measurement results, a correlation was obtained as

$$
\frac{\delta}{D_{i}}=\left\{\begin{array}{cc}
\frac{0.67 C a^{2 / 3}}{1+3.13 C a^{2 / 3}+0.504 C a^{0.672} R e^{0.589}-0.352 W e^{0.629}} & (R \mathrm{e}<2000) \\
\frac{106.0\left(\frac{\mu^{2}}{\rho \sigma} \frac{1}{D_{i}}\right)^{\frac{2}{3}}}{1+497.0\left(\frac{\mu^{2}}{\rho \sigma} \frac{1}{D_{i}}\right)^{\frac{2}{3}}+7330\left(\frac{\mu^{2}}{\rho \sigma} \frac{1}{D_{i}}\right)^{0.672}-5000\left(\frac{\mu^{2}}{\rho \sigma} \frac{1}{D_{i}}\right)^{0.629}} & (R \mathrm{e}>2000)
\end{array}\right.
$$

With regard to microchannel boiling, bubbles nucleate and quickly grow to the channel size such that elongated bubbles are formed; these are confined by the channel walls and grow nonlinearly in length owing to the rapid evaporation of the microlayer (see Fig. 1 by Utaka et al. [6]). This requires using the full Navier-Stokes equations rather than the Stokes equations or steady Navier-Stokes equations that neglect local acceleration. However, there are not sufficient published studies on microlayer formation under accelerated motion [4].

Moriyama and Inoue [7] reported that the microlayer thickness follows one of two trends as the interface traveling velocity increases. They considered that a transition in the controlling mechanism occurred in the range of their experimental conditions. Two regimes for microlayer formation in their experimental study were identified according to whether the Bond number, $B o=\frac{\rho D_{i}^{2}}{\sigma}\left(\frac{U^{2}}{2 D}\right)$ (which is calculated using the approximately constant acceleration of interface movement rather than gravity) is greater than 2 as Eq. (3),

$$
\frac{\delta}{s}= \begin{cases}0.07 C a^{0.41} & \\ 0.10\left[\frac{1}{s} \sqrt{\frac{\mu_{L} t_{g}}{\rho_{L}}}\right]^{0.84} & (B o \leq 2) \\ & (B o>2)\end{cases}
$$


where $s$ is the distance between the two parallel plates; $D$ is the distance from the bubble forefront to the incipit site; $t_{g}$ is the bubble growth time; and $\mu_{L}$ and $\rho_{L}$ are the viscosity and density of the liquid, respectively. Han and Shikazono [8] assumed that under accelerated conditions, the bubble nose curvature is affected by the viscous boundary layer. Based on the measured results of the microlayer thickness under adiabatic conditions affected by the acceleration for the circular tube, a modification coefficient $f=0.692 B o^{0.414}$ was obtained by least-squares fitting of the data at Bo $>1$. Then, a correlation using the Bond number, Bo, for the data at $B o>1$ is proposed as

$$
\frac{\delta}{D}=\frac{0.968 \mathrm{Ca}^{2 / 3} \mathrm{Bo}^{-0.414}}{1+4.838 \mathrm{Ca}^{2 / 3} \mathrm{Bo}^{-0.414}}
$$

Based on a review of the literature, an insufficient number of studies have examined the accelerated motion of bubbles confined in mini-/microchannels with respect to boiling when the bubble grows nonlinearly due to rapid evaporation of the microlayer. In the present study, to clarify the formation mechanism of the microlayer, the initial microlayer thickness-which is defined as the thickness of the microlayer just after the bubble tip at the location at which the interface between the vapor and the liquid is approximately parallel to the heating plate, as shown in Fig. 2-was measured directly using a laser extinction method for the test fluid of water, ethanol, toluene, and HFE7200. In addition, the progress of bubble growth in two dimensions with acceleration was also simulated by the volume of fluid (VOF) method using the computational fluid dynamics (CFD) package of FLUENT12.1. Furthermore, a nondimensional correlation was obtained based on the measurement data and numerical studies.

\section{NOMENCLATURE}

$\begin{array}{lll}A & \left(\mathrm{~m}^{\mathrm{c}}\right) & \text { extinction coefficient } \\ a & \left(\mathrm{~m} / \mathrm{s}^{2}\right) & \text { acceleration } \\ D & (\mathrm{~mm}) & \text { distance from bubble inception site } \\ I & & \text { laser intensity } \\ I_{0} & & \text { incident laser intensity } \\ s & (\mathrm{~mm}) & \text { gap size } \\ V_{L} & (\mathrm{~m} / \mathrm{s}) & \text { local bubble forefront velocity } \\ \delta & (\mu \mathrm{m}) & \text { microlayer thickness }\end{array}$


$(\mu \mathrm{m}) \quad$ initial microlayer thickness

$\delta_{0 n} \quad$ nondimensional initial microlayer thickness

$$
\delta_{0 n}=\delta_{0} / s
$$

$\delta_{V} \quad(\mu \mathrm{m}) \quad$ viscous boundary layer thickness

$\mu \quad(\mathrm{Pa} \cdot \mathrm{s}) \quad$ viscosity

$\sigma \quad(\mathrm{N} / \mathrm{m}) \quad$ surface tension coefficient

$\rho \quad\left(\mathrm{kg} / \mathrm{m}^{3}\right) \quad$ density

Ca

Capillary number $\frac{\mu_{L} V_{L}}{\sigma}$

We

Weber number $\frac{\rho s V_{L}^{2}}{\sigma}$

Bo

Bond number $\frac{\rho s^{2} a}{\sigma}$

\section{EXPERIMENT}

\subsection{Experiment apparatus and procedure}

A schematic diagram of the experimental apparatus is shown in Fig. 3(a). The vapor generator was located between a heliumneon (He-Ne) infrared laser emitter with a wavelength of $3.39 \mu \mathrm{m}$ and a lead-selenium ( $\mathrm{Pb}-\mathrm{Se})$ detector. A liquid reservoir and a heating tank were placed upstream in the microchannel test apparatus. The cross-sectional area of the liquid reservoir was sufficiently large to maintain a constant liquid level in the microchannel, which could ensure that it was close to the pool boiling in the microchannel but not flow boiling. The liquid supplied to the microchannel apparatus was boiled in a heating tank to exclude noncondensing gas and retain the temperature of the liquid to be saturated. An orifice on the inlet of the microchannel was used to restrain the fluctuation of the liquid flow rate caused by the bubbles' growth and collapse. Vapor generated from the microchannel vapor generator flowed through a condenser and back to the reservoir. Figure 3(b) shows the details of the microchannel test apparatus. Quartz glass with a high transparency for infrared light was mainly utilized for the test apparatus to enable more accurate measurements. The channel thickness between two parallel quartz glass plates has a variable thickness by altering the spacers. Three different gap sizes of $0.15,0.30$, and $0.50 \mathrm{~mm}$ were adopted in the present study. The real gap size of the microchannel, as measured with a plastic gauge, was in the range of $0.147-0.158 \mathrm{~mm}$ for a $0.15-\mathrm{mm}$ test gap size. Therefore, it was confirmed that the gap size was constant and sufficiently accurate with respect to the target value, as reported by Utaka et al. [6]. Passages for high-temperature air used as the heating source to heat the microchannel were positioned at the back and front of the microchannel. The central part of 
the 82-mm-high passage, which essentially served as the heating area, was narrowed to enhance heating. The width of the passage was $45 \mathrm{~mm}$. The heat flux into the microchannel was controlled by varying the air temperature. The principle of the laser extinction method was used to determine the microlayer thickness by using Lambert’s law, as shown in Eq. (5).

$$
\delta=-\left(\frac{1}{A}\right) \ln \left(I / I_{0}\right)
$$

where $\delta$ denotes the thickness of the microlayer through which the laser travels. In this experiment, because the bubble formed symmetrically in the microchannel, the initial microlayer thickness $\delta_{0}$ cited herein is calculated as half the measured thickness. Regarding the experiment, this refers to the first signal appearing on the laser ray detector when the bubble passed through the point where the laser ray passed through the microchannel, which was reported by Utaka et al. [6]. Here, A represents the extinction coefficient, as shown in Table 1. The light intensity at the detector when the microchannel being measured is filled with steam is denoted by $I_{0}$, and the light intensity when the microchannel is filled with both a thin liquid layer and steam is denoted by $I$. The laser ray was focused to a diameter of $0.6 \mathrm{~mm}$ at the microchannel using a convex lens. To reduce the effect due to the instability of the laser emitter, the laser beam was divided by the beam splitter, and then the reference light intensity could be measured simultaneously. This light extension method is sufficiently accurate for measuring the microlayer thickness on a micron scale, and a detailed investigation on its measurement precision was carried out by Zhang [9].

\subsection{Measurement results}

The microlayer forms as a result of liquid remaining on the heating surface immediately after the bulk liquid is pushed away by the bubble growth. The microlayer thickness varies with the effects of the bubble growth rate. In the present study, attention was focused on the initial microlayer thickness $\delta_{0}$, which determines the basic characteristics of heat transfer in the microchannel [6]. The variation of the initial microlayer thickness versus the bubble forefront velocity is shown in Fig. 4 for HFE7200, toluene, water, and ethanol for three different microchannel gap sizes of $0.50,0.30$, and $0.15 \mathrm{~mm}$. The initial microlayer thickness was strongly affected by the gap size and was increased with the increasing gap size. For ethanol, there was no result in the present study on the gap size except for the minimum size of $0.15 \mathrm{~mm}$. This was because of the large extinction coefficient for the laser used in the present experiment. Moreover, the results for any of the four liquids showed a similar tendency for any gap size — that is, in low-velocity regions, the microlayer thickness increases with the forefront velocity; however, as the velocity increases, the thickness becomes almost constant or decreases slightly for sufficiently high bubble forefront velocities.

For the four kinds of liquids with a representative gap size, figure 5 shows the variations of the bubble forefront velocity against the microlayer thickness with $D$ (the distance from the bubble inception site) as a parameter. It could be observed that in the region of 
high velocity, the initial microlayer thickness increased with increasing $D$, whereas in the region of low velocity, the effect of $D$ became weak or vanished.

\subsection{Comparison between present results and other measurements}

Because of the limited data on the microlayer thickness measurements during boiling in the microchannel, there are only a few cases for which to compare the independent data with various methods. Figure 6 shows experimental data of the initial microlayer thickness for HFE7200 with the calculated thickness for R113 from Moriyama and Inoue [7] and Han and Shikazono [8] for FC40 (all

three kinds of liquids have largely similar physical properties, as shown in Table 2). Although the experimental conditions are not exactly the same, the microlayer thicknesses measured by Moriyama and Inoue [7] are significantly (several times) thinner compared with the measurements of the present research and those of Han and Shikazono [8]. Furthermore, Fig. 7 shows the measurements of water with a channel size of $0.5 \mathrm{~mm}$ in the present study for a parallel plate channel and Han and Shikazono [8] for a circular tube. In the region of low velocity, the measurements obtained by the two different methods show good agreement; however, perhaps due to the different channel geometry and experimental conditions (for example, the acceleration of the vapor forefront, the effect of which is significant in the high-velocity region but weak in the low-velocity region), the data at the region of high velocity are in slight disagreement. It could be confirmed that the methods of Han and Shikazono [8] and those used in the present study result in similar accuracy of the microlayer thickness measurements.

\section{NUMERICAL SIMULATION OF BUBBLE GROWTH PROCESS IN MICROCHANNEL}

CFD technology was used for simulating the hydrodynamic characteristics of the bubble growth process in the microchannel. One of the main merits of CFD is that it can provide deeper insight into the underlying physical mechanisms and thus foster understanding of the gas-liquid two-phase flow. It can also provide a unique velocity field, phase distribution, and microlayer with high spatial and temporal resolution, which is difficult to do precisely, even with the current most advanced experimental techniques.

\subsection{Formulation of the problem and the solution strategy}

The CFD software FLUENT (Release 12.12010 ) was used to simulate the growth process of a single bubble in an initially static liquid in a microchannel within two parallel plates. In FLUENT, the control volume method—sometimes referred to as the finite volume method-is used to discretize the transport equations. The movement of the gas-liquid interface is tracked based on the distribution of $\alpha_{G}$, the volume fraction of gas in a computational cell, where $\alpha_{v}=0$ in the liquid phase and $\alpha_{v}=1$ in the gas phase. Therefore, the gas-liquid interface exists in the cell where $\alpha_{v}$ lies between 0 and 1 . The geometric reconstruction scheme that is based 
on the piece linear interface calculation (PLIC) method of Youngs [10] is applied to reconstruct the bubble-free surface. The surface tension is approximated by the continuum surface force model of Brackbill et al. [11].

The continuity equation and a single momentum equation shown in Eq. (6) are solved throughout the domain, and the resulting velocity field is shared among the phases. The momentum equation is dependent on the volume fractions of all phases through the properties $\rho$ and $\mu$,

$$
\frac{\partial}{\partial t}(\rho)+\nabla \llbracket(\rho \vec{v})=0
$$

The momentum equation

$$
\frac{\partial}{\partial t}(\rho \vec{v})+\nabla \square(\rho \vec{v} \vec{v})=-\nabla p+\nabla\left[\mu\left(\nabla \vec{v}+\nabla \vec{v}^{T}\right)\right]+\vec{F}_{S F}
$$

where $\overrightarrow{F_{S F}}$ is a continuum surface tension vector.

The tracking of the interface between the gas and liquid is accomplished by the solution of a continuity equation for the volume fraction of gas.

$$
\frac{\partial}{\partial t}\left(\alpha_{v}\right)+\vec{v} \square \nabla \alpha_{v}=0
$$

The volume fraction equation will not be solved for the liquid; the liquid volume fraction will be computed based on the following constraint:

$$
\alpha_{v}+\alpha_{l}=1
$$

The surface tension model in FLUENT is the continuum surface force (CSF) model proposed by Brackbill et al. [11]. With this model, the addition of surface tension to the VOF calculation results in a source term in the momentum equation, as shown in Eq. (7).

Thus, the source term, $\vec{F}_{S F}$, in the momentum equation can be specified as 


$$
\overrightarrow{F_{S F}}=\sigma k \hat{n}\left[\frac{\alpha_{l} \rho_{l}+\alpha_{\mathrm{v}} \rho_{\mathrm{v}}}{0.5\left(\rho_{l}+\rho_{\mathrm{v}}\right)}\right]
$$

where $\sigma$ is the surface tension coefficient, and $k$ is the free-surface curvature defined in terms of the divergence of the unit normal, $\hat{n}$, as:

$$
k=\nabla \hat{n}=\frac{1}{|n|}\left[\left(\frac{n}{|n|} \mid \nabla\right)|n|-(\nabla \square n)\right]
$$

where

$$
\hat{n}=\frac{n}{|n|}, \quad n=\nabla \alpha_{v}
$$

The properties of liquid or gas are used in the transport equations when the computational cell is in the liquid or gas phase, respectively. When it is in the interface between the gas and liquid phases, the density and viscosity in each cell are given by

$$
\begin{aligned}
& \rho=\alpha_{v} \rho_{v}+\left(1-\alpha_{v}\right) \rho_{l} \\
& \mu=\alpha_{v} \mu_{v}+\left(1-\alpha_{v}\right) \mu_{l}
\end{aligned}
$$

\subsection{Model geometry and initial and boundary conditions}

Because of the large aspect ratio of the cross section of the microchannel used in this study, the fluid flow inside the microchannel can be considered a two-dimensional flow. Therefore, a two-dimensional coordinate system assuming symmetry about the centerline of the microchannel was used. To investigate the effect of acceleration or distance from the bubble forefront interface to the bubble inception site, the calculation domain was determined to be $100 \mathrm{~s}$, where $s$ is the microchannel gap size. Figure 8 displays the boundary conditions used in the simulation. The no-slip wall condition is applied. Using a user-defined function, the velocity normal to the outlet is specified as unsteady flow $u_{\text {out }}=a \cdot t$, where $a$ is the acceleration of the bubble forefront movement, and $t$ is the time of bubble growth, as shown in Fig. 8. The inlet for liquid and gas is specified as a pressure-fixed boundary condition. Then, the process of elongated bubble growth is simulated under initial conditions [Eq. (15)] and boundary conditions [Eq. (16)]. 


$$
\begin{aligned}
& \begin{array}{l}
u=v=0 \\
\delta_{0, t=0}=L_{y}-R_{b}
\end{array} \\
& \text { Bubble }_{t=0} \in\left[\left(0 \leq x \leq L_{b}-R_{b}, 0 \leq y \leq R_{b}\right) \cup\left(L_{b}-R_{b} \leq x \leq L_{b}, 0 \leq y \leq \sqrt{R_{b}^{2}-x^{2}}\right)\right] \\
& \qquad \begin{aligned}
y & =L_{y}: u=0, v=0 \\
y & =0: \frac{\partial u}{\partial y}=0, \frac{\partial v}{\partial y}=0 \\
x & =0: \frac{\partial u}{\partial x}=0, \frac{\partial v}{\partial y}=0, p=p_{0} \\
x & =L_{x}: u=a \llbracket
\end{aligned}
\end{aligned}
$$

\subsection{Studies of grid independence and initial condition}

Four different grid sizes ranging from 1 to $5 \mu \mathrm{m}$ in the microchannel length of $10 \mathrm{~mm}$ were used to calculate the bubble shape for the same initial condition, as shown in Fig. 9(a). The grid size of $5 \mu \mathrm{m}$ was apparently too large to accurately capture the interface position compared with the other three sizes. Using the grid sizes of 1, 2, and $2.5 \mu \mathrm{m}$, almost the same results were obtained—namely, the result is grid-independent. Therefore, the grid of $2.5 \mu \mathrm{m}$ was used to calculate the bubble growth in this study. Then, there were almost more than four elements in the field of the microlayer, which is considered sufficient to accurately capture the microlayer.

Figure 9(b) shows the plot of the bubble shape calculated from three different initial conditions of microlayer thicknesses (8, 10, and $15 \mu \mathrm{m})$ at $t=0$ but with the same acceleration and local velocity of the bubble forefront. The local bubble forefront $V_{L}$ is defined as the velocity of the liquid-gas interface at the centerline. No effect can be observed from the results after the bubble growth to longer than approximately $2 \mathrm{~mm}$. In this study, $10 \mu \mathrm{m}$ was adopted as the initial condition of the microlayer thickness in all cases, which will not affect the simulation results.

\subsection{Comparison between simulation and measurement results for HFE7200}

HFE7200 was adopted as a representative test fluid to investigate the outcome of each parametric effect of the physical properties. In the low-velocity region, the experimental results show that the microlayer thickness almost increases linearly with the bubble forefront velocity. This is because the velocity profile is similar to that of the steady internal flow. The viscous boundary layer is still not developed, and its thickness $\delta_{V}$ is much thicker than the thickness of the microlayer $\delta_{0}$-i.e., the viscous boundary layer has little effect on the development of the microlayer, as shown in Fig. 10(a) $\left(\delta_{0} / \delta_{V}=0.26\right)$. In this region, the distance from the bubble 
inception site to the bubble forefront $D$ (or acceleration $a$, according to the relationship $a=V_{L}{ }^{2} / 2 D$ by assuming that the acceleration is approximately constant) almost does not affect the buildup of the microlayer.

The experimental results show that in the region of high velocity, the microlayer thickness increased with $D$, as shown in Figs. 4 and 5. This can be limited by the viscous boundary layer when it develops well in the bulk of the liquid in front of the bubble forefront and its thickness $\delta_{V}$ approaches the thickness of microlayer $\delta_{0}$, as indicated by Moriyama and Inoue [7]. The velocity gradient increases near the wall, and the profile of the velocity for the core of the fluid nearly becomes a parallel flow instead of parabolic, as shown in Fig.10(b), and the ratio of $\delta_{0} / \delta_{V}$ increases. Figure 11 shows the comparison between the simulations and measurements for different accelerations and velocities—nearly good agreement is achieved, except the slope of the linearly increasing region is slightly smaller than that of the measurement. The ratio of the microlayer thickness $\delta_{0}$ to the boundary layer thickness $\delta_{V}$ obtained by the simulation results of HFE7200 for different accelerations and velocities is shown in Fig. 12. The ratio shows an obvious increasing trend with the velocity in the low-velocity region, but the increasing trend weakens for the high-velocity region. Therefore, it is clear that the microlayer thickness formation is hardly affected by the viscous boundary layer in the low-velocity region but is limited by $\delta_{V}$ in the high-velocity region, as stated previously.

\subsection{Study of effect of physical properties}

Figures 13(a)-(d) show the effects of the physical properties, such as surface tension, viscosity and density on the thickness of the microlayer, and the shape of the bubble tip. For microchannel boiling, the surface tension coefficient is a dominant parameter. This pushes out the liquid in the microlayer by the inner pressure of the vapor bubble due to the very large curvature of the vapor-liquid interface at the bubble forefront. Two simulation results of the bubble nose shape for different surface tension coefficients are shown

in Fig. 13(a). The bubble forefront indicated by the blue line that is calculated with relatively small surface tension $9.4 \mathrm{mN} / \mathrm{m}$ is sharper than that with large surface tension of $58.9 \mathrm{mN} / \mathrm{m}$; consequently, the thickness of the microlayer in the flat region for the bubble with small surface tension is thicker than the one with a large surface tension coefficient.

On the contrary, viscous force is favorable to the microlayer, and large viscosity increases the thickness, as shown in Fig. 13(b). The shape of the bubble calculated with small viscosity is more blunt than the bubble with large viscosity because its weak viscous force cannot overcome the surface tension force (which acts to minimize the surface areas) and cannot remain as liquid as the one with large viscosity. Consequently, the thickness of the microlayer for the bubble with weak viscosity is relatively thin. 
It was stated in the introduction that for steady slow motion, the microlayer thickness is determined by the balance between the surface tension and viscous force; therefore, the microlayer thickness is a function of the capillary number, and the inertial force can almost be omitted. However, as the bubble movement velocity increased, the inertial force began to affect the formation of the microlayer and increase its thickness. The simulation results of the bubble forefront shape for two different densities at low velocity are shown in Fig. 13(c). Because the forefront velocity is not high, the two bubble shapes close to each other-i.e., the thickness of the flat microlayer region-are almost the same, although the nose of the bubble with a large density is slightly elongated due to the inertial force. When the velocity increases, as shown in Fig. 13(d), the effect of the inertial force becomes significant, and larger density increases the microlayer thickness, as mentioned previously.

\section{DIMENSION ANALYSIS AND CORRELATION}

To create a correlation, dimension analysis was attempted, as shown in the following. Based on the experiment, it was shown that the microlayer thickness can be expressed as

$$
\delta_{0} \square f\left(V_{L}, D, s, \rho, \mu, \sigma\right)
$$

The experimental and simulation results show that the microlayer thickness is proportional to viscosity and density and inverse proportional with surface tension. Therefore, the thickness can be expressed as

$$
\delta_{0} \sim \frac{\mu \rho}{\sigma} \sim \frac{M \tau}{L^{4}}
$$

where $M, L$, and $\tau$ denote mass, length, and time, respectively. By introducing $V_{L}$ and $s$ to which the microlayer thickness is also proportional, according to the experimental results, the right side of Eq. (18) can be nondimensionalized as

$$
\frac{\rho \mu V_{L}^{3} s}{\sigma^{2}} \sim 1
$$

Using the Buckingham $\Pi$ theorem, we can also determine that the nondimensional microlayer thickness is qualitatively a function of

$\mathrm{Ca}$, We, and $\mathrm{Bo}$, where $\mathrm{Bo}=\frac{\rho a s^{2}}{\sigma}$ is the Bond number that represents the ratio of the inertial force due to the acceleration to the surface tension force. $W e=\rho u^{2} s / \sigma$ is the Weber number that represents the ratio of the inertial force to the surface tension force. Here, according to the study of Moriyama and Inoue [8], as the velocity increases, the microlayer is limited by the viscous boundary layer. Therefore, perhaps it is reasonable to nondimensionalize the microlayer thickness with the viscous boundary layer thickness, and then the nondimensional relationship changes to be

$$
\frac{\delta_{0}}{\delta_{V}} \sim \frac{\rho \mu V_{L}^{3} S}{\sigma^{2}}
$$


Here, although in the low-velocity region, formation of a thin liquid film is not considered to be strongly dependent on the boundary layer because of the accelerative growth of the elongated bubble during the microchannel boiling phenomenon, as mentioned previously, it is thought that the influence of the forces of inertia and acceleration in addition to viscosity and surface tension for thin liquid film thickness formation should be considered. The effects of acceleration, surface tension, and viscosity are included in Eq.

(20). If the boundary layer thickness is transformed as

$$
\delta_{V n}=\frac{\delta_{V}}{s}=\sqrt{\frac{D V_{L}}{\rho V_{L} s^{2}}}=\sqrt{\frac{2 D \sigma}{\rho V_{L}^{2} s^{2}} \cdot \frac{\mu V_{L}}{2 \sigma}}=\sqrt{\frac{1}{2}} C a^{0.5} B o^{-0.5}
$$

Equation (20) is transformed into Eq. (21) using nondimensional numbers.

$$
\sqrt{2} \frac{\delta_{0}}{S} C a^{-0.5} \mathrm{Bo}^{0.5} \sim \mathrm{Ca} \cdot \mathrm{We}
$$

All of the experimental data are plotted in Figs. 14 and 15, where the coordinate is the nondimensional group of Eq. (22). Figures 14(a)-(d) show the effect of the test fluid; each graph denotes each fluid brought to the front, respectively, because the symbols of the front fluid tend to overlap other data. In addition, Figs. 15(a)-(c) show the effect of each gap size. This figure shows that each fluid and each gap size correlated well without apparent discord, as shown in Figs. 14 and 15, respectively. Equations (23) and (24) are fitting equations for the experiment data with $C a \cdot W e<0.1$ and $C a \cdot W e>10$, respectively, plotted as solid lines in Fig. 14 , and the region between the two regions is considered the transition region. Then, the continuous equation can be created by combining the two equations obtained from Fig. 14, as shown in Eq. (25) (dashed line in Fig. 14).

$$
\begin{gathered}
\frac{\delta_{0}}{S} C a^{-0.50} \mathrm{Bo}^{0.50}=0.32(\mathrm{Ca} \cdot \mathrm{We})^{0.45} \\
\frac{\delta_{0}}{s} C a^{-0.50} \mathrm{Bo}^{0.50}=0.44(\mathrm{Ca} \cdot \mathrm{We})^{0.03} \\
\frac{\delta_{0}}{S} \mathrm{Ca}^{-0.5} \mathrm{Bo}^{0.50}=\left\{\left[0.32(\mathrm{Ca} \cdot \mathrm{We})^{0.45}\right]^{-3}+\left[0.44(\mathrm{Ca} \cdot \mathrm{We})^{0.03}\right]^{-3}\right\}^{-1 / 3}
\end{gathered}
$$

Then, Eq. (25) can be rewritten in a simpler form as Eq. (26).

$$
\frac{\delta_{0}}{s}=\left\{\left[0.32 C a^{0.95} W e^{0.45} B o^{-0.5}\right]^{-3}+\left[0.44 C a^{0.53} W e^{0.03} \mathrm{Bo}^{-0.5}\right]^{-3}\right\}^{-1 / 3}
$$

To confirm the accuracy of Eq. (26) in detail, the experimental data were first divided into several groups based on a certain range of the Weber number; then, the data with a fixed range of We were divided into subgroups based on a corresponding range of the Bond number. The correlation of Eq. (26) was plotted in Fig.16, and the correlations based on the upper and lower boundary values of We are plotted in dashed and solid lines in red or black corresponding to the boundary value of Bo. The experimental data in each range of 
the Weber number and Bond number are in relatively good agreement with the correlation of Eq. (26) derived from dimension analysis.

\section{CONCLUSIONS}

Using the laser extinction method, experiments were performed to directly measure the microlayer that forms on a heating surface by vapor growth during boiling in a mini-/microchannel formed by two parallel quartz glass plates. The process of bubble growth with acceleration was simulated in two-dimensional microchannels using the VOF method. Based on the results of the experiment and simulation, a correlation equation was created using dimension analysis. The conclusions of this study are as follows:

(1) The initial microlayer thickness was mainly determined by the gap sizes and the velocity of the bubble forefront. The effect of the distance from the bubble inception site to the bubble forefront became significant with the increase of the bubble velocity.

(2) The trend in the variation of the microlayer thickness relative to the velocity of the bubble forefront changed with the velocity beyond certain values. In the region of low velocity, the microlayer thickness increased with increasing velocity, while in the region of high velocity, the microlayer thickness slightly decreased with increasing velocity.

(3) The microlayer thickness calculated from the simulation results showed good agreement with the experiment.

(4) In the low-velocity region, the simulation showed that the velocity profile of the liquid in front of the bubble forefront is parabolic; therefore, the development of the microlayer is not affected by the boundary layer-i.e., the effect of distance or acceleration is weak at this region. In the high-velocity region, the core flow of liquid in front of the bubble forefront became a parallel flow, which is similar to the inlet of internal flow. Consequently, the viscous boundary layer thinned and limited the development of the microlayer.

(5) The numerical results revealed that the microlayer thickness increases in proportion to density and viscosity and is inversely proportionate to the surface tension coefficient.

(6) On the basis of dimension analysis and the results of the experiments, a uniform empirical correlation in parallel mini/microchannels was proposed for the nondimensional microlayer thickness $\delta_{0} / \mathrm{s}$ correlated well as a function of the Capillary number, Weber number, and Bond number. The present correlation predicts the experimental results within an accepted error range.

\section{REFERENCES}

[1] Taylor, G.I., Deposition of a viscous fluid on the wall of a tube, Journal of Fluid Mechanics 10 (1961) 1161-1165.

[2] Bretherton, F.P., The motion of long bubbles in tubes, Journal of Fluid Mechanics 10 (1961) 166-188.

[3] Aussillous, P., and Quere, D., Quick deposition of a fluid on the wall of a tube, Physics of Fluids 12 (2000) $2367-2371$. 
[4] Zhang, Y.H., and Utaka, Y., Characteristics of microlayer formation and heat transfer in mini/microchannel boiling systems: A review, Frontiers in Heat and Mass Transfer 3 No. 1 (2012) 013003.

[5] Han, Y., and Shikazono, N., Measurement of the liquid film thickness in micro tube slug flow, International Journal of Heat and Fluid Flow 30 No. 5 (2009) 842-853.

[6] Utaka, Y., Okuda, S., and Tasaki, Y., Configuration of the micro-layer and characteristics of heat transfer in a narrow gap mini/micro-channel boiling system, International Journal of Heat and Mass Transfer 52 (2009) 2205-2214.

[7] Moriyama, K., and Inoue, A., Thickness of the liquid film formed by a growing bubble in a narrow gap between two horizontal plates, Trans. ASME, Journal of Heat Transfer 118 (1996) 132-139.

[8] Han, Y., and Shikazono, N., The effect of bubble acceleration on the liquid film thickness in micro tubes, International Jouranal of Heat and Fluid Flow 31 (2010) 630-639.

[9] Zhang, Y.H., Formation mechanism and characteristics of microlayer between elongated bubble and heating surface in parallel microchannel boiling, Doctoral Thesis (2012) Yokohama National University.

[10] Youngs, D.L., Time-dependent multi-material flow with large fluid distortion, in: Morton, K.W., and Baibnes, M.J. (Eds.), Numerical Methods for Fluid Dynamics, Academic Press, New York (1982) 273.

[11] Brackbill, J.U., Kothe, D.B., and Zemach, C., A continuum method for modeling surface tension, Journal of Computational Physics 100 (1992) 335-354. 
Table 1 Extinction coefficient of the test fluids

Table 2 Properties of the test fluids at atmospheric pressure and saturation point

Fig. 1 Velocity of bubble forefront increasing with distance from bubble site by Utaka et al. [6]

Fig. 2 Schematic diagram of elongated bubble in microchannel

Fig. 3 Experimental apparatus for measuring microlayer thickness: (a) schematic of entire experimental system; (b) details of minichannel and heat transfer plate

Fig. 4 Microlayer thicknesses versus local bubble forefront velocity for water, toluene, and HFE7200

Fig. 5 Effect of distance from incipient bubble site $D$

Fig. 6 Comparison of initial microlayer thickness measured by different methods for refrigerants

Fig. 7 Comparison of microlayer thickness of water with channel size of $0.5 \mathrm{~mm}$ from present study and study by Han and Shikazono [8]

Fig. 8 Schematic representation of the geometry and boundary conditions used in the simulations

Fig. 9 Confirmation of calculation conditions: (a) comparison of bubble shapes for different sizes of meshes; (b) effect of initial condition of microlayer thicknesses

Fig. 10 Velocity profile of liquid bulk in front of bubble forefront for HFE7200 of gap width of $0.3 \mathrm{~mm}:(\mathrm{a}) a=60 \mathrm{~m} / \mathrm{s}^{2}, V_{L}=0.74$ $\mathrm{m} / \mathrm{s}$, and $\delta_{0} / \delta_{V}=0.26$; (b) $a=100 \mathrm{~m} / \mathrm{s}^{2}, V_{L}=1.95 \mathrm{~m} / \mathrm{s}$, and $\delta_{0} / \delta_{V}=0.83$

Fig. 11 Comparison between simulation and measurement results for HFE7200 with $s=0.3$ for fixed distances from bubble inception site $D$

Fig. 12 Ratio of microlayer thickness to boundary layer thickness obtained by simulation results of HFE7200 for different accelerations and velocities

Fig. 13 Parametric effects of physical properties: (a) effect of surface tension; (b) effect of viscosity; (c) effect of density for low velocity; and (d) effect of density at high-velocity region

Fig. 14 Relation among nondimensional microlayer thickness and dominant nondimensional parameters (effect of test fluids): (a) HFE7200; (b) toluene; (c) water; and (d) ethanol

Fig. 15 Relation among nondimensional microlayer thickness and dominant nondimensional parameters (effect of gap size): (a) $s=$ $0.15 \mathrm{~mm}$; (b) $s=0.30 \mathrm{~mm}$; and (c) $s=0.50 \mathrm{~mm}$

Fig. 16 Comparison between correlations and experiments 


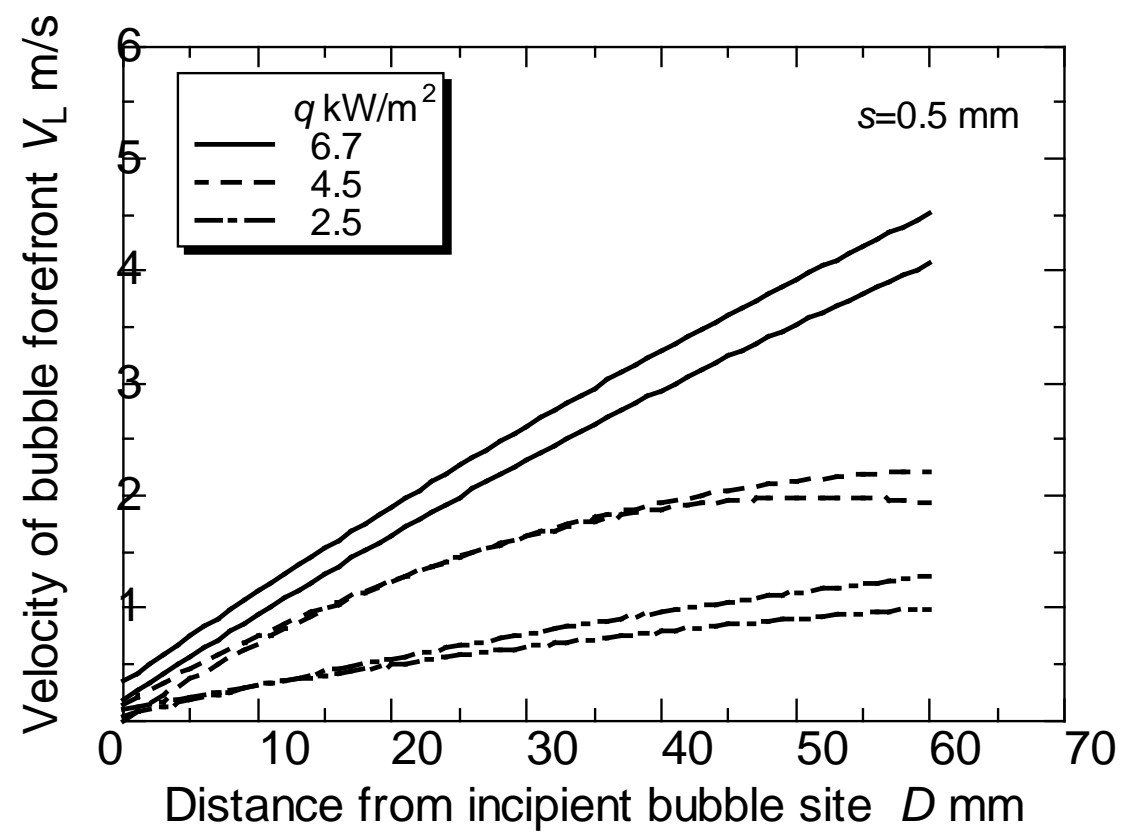

Fig. 1 


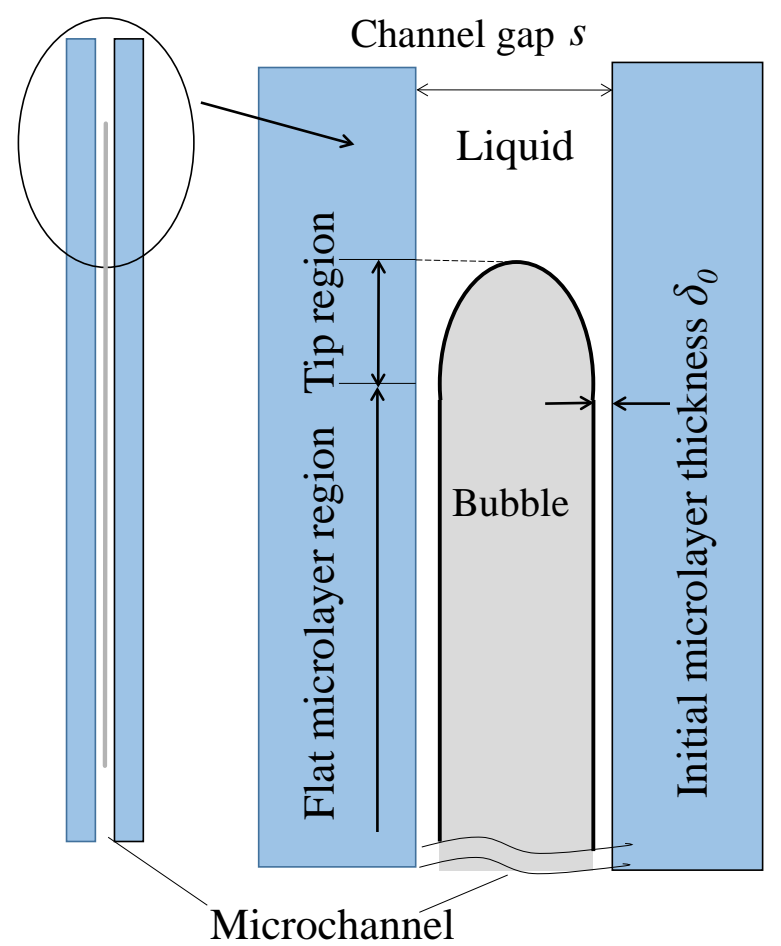

Fig. 2

Table 1

\begin{tabular}{ccccc}
\hline & Water & Toluene & HFE7200 & Ethanol \\
\hline$A\left(\mathrm{~m}^{-1}\right)$ & $5.42 \times 10^{4}$ & $3.15 \times 10^{4}$ & $1.88 \times 10^{4}$ & $1.09 \times 10^{5}$ \\
\hline
\end{tabular}




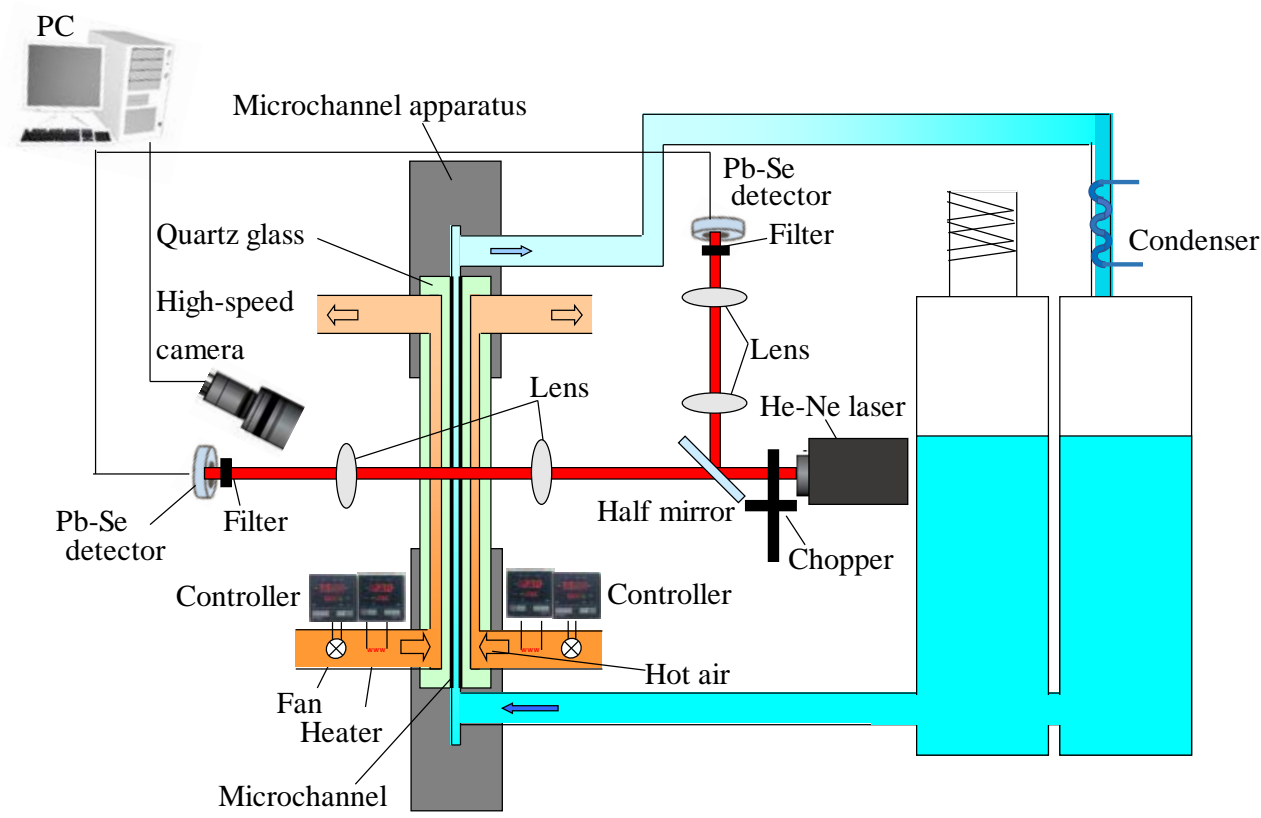

(a)

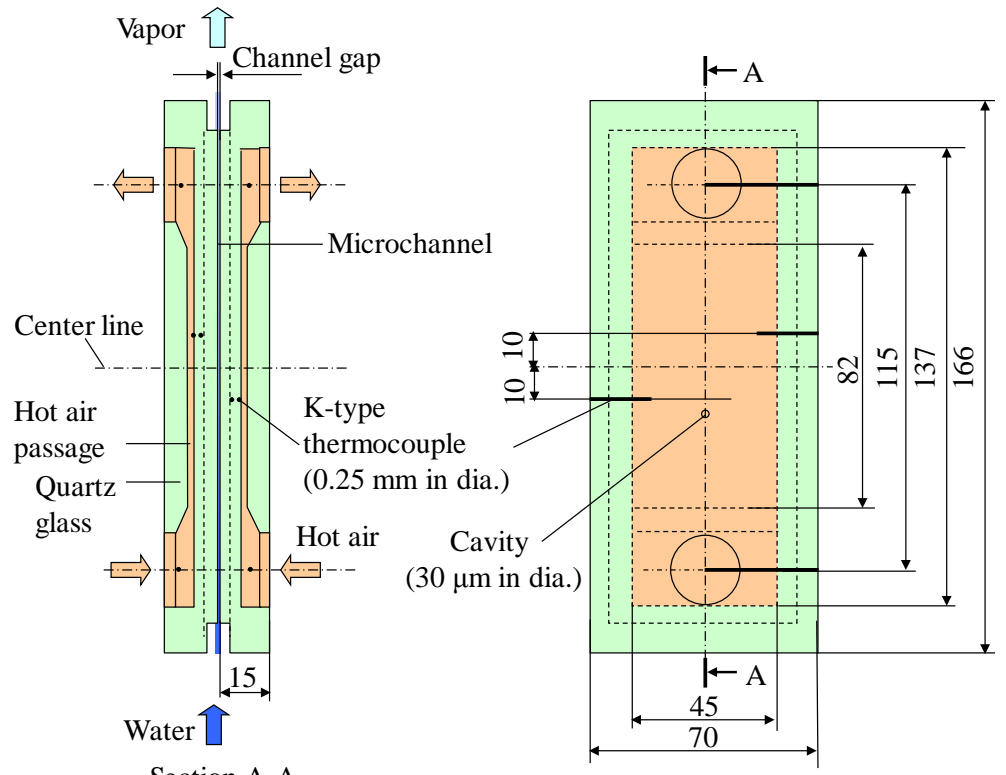

Section A-A

Quartz glass plates of micro-channel

(b)

Fig. 3 


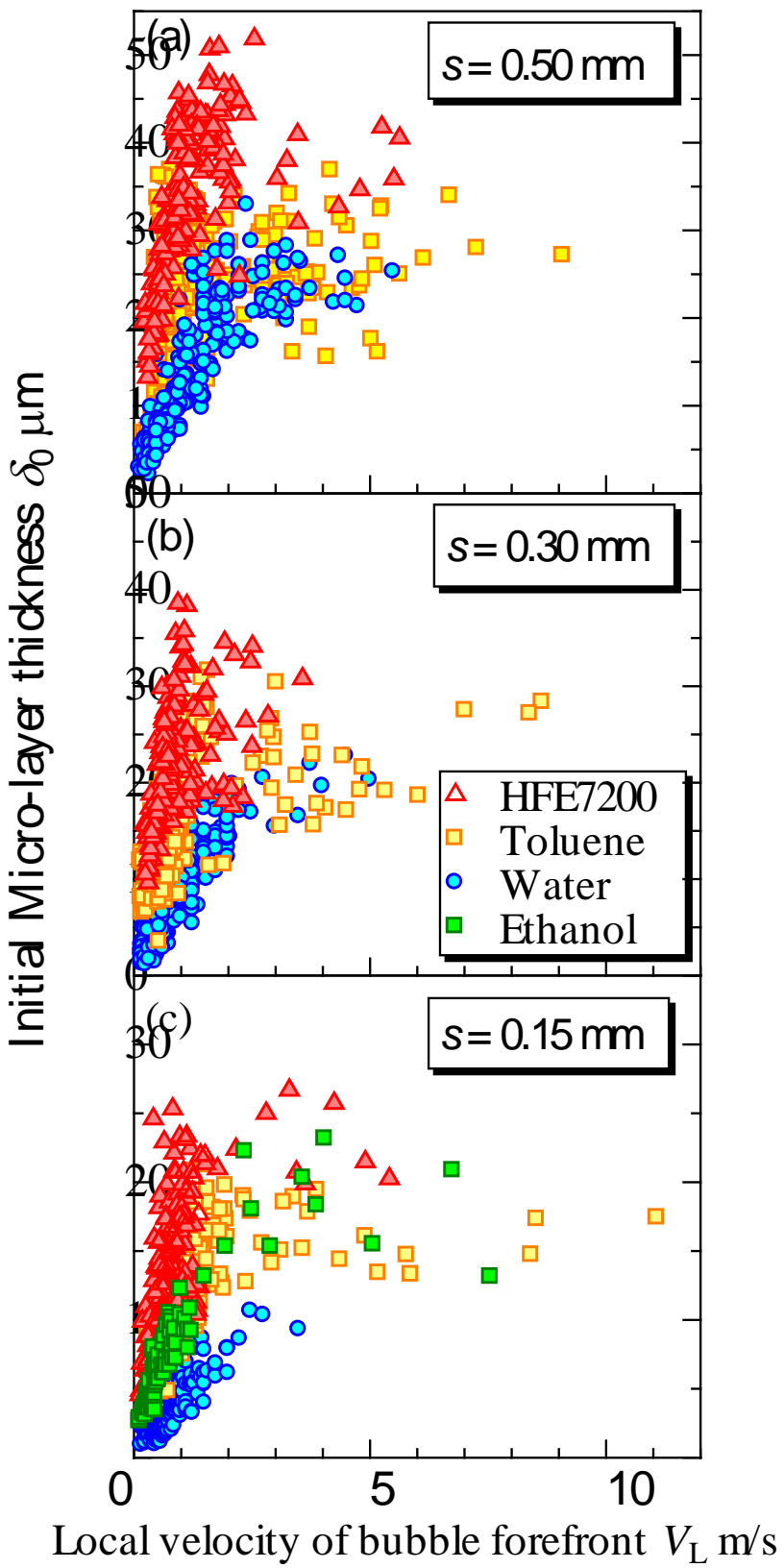

Fig. 4 


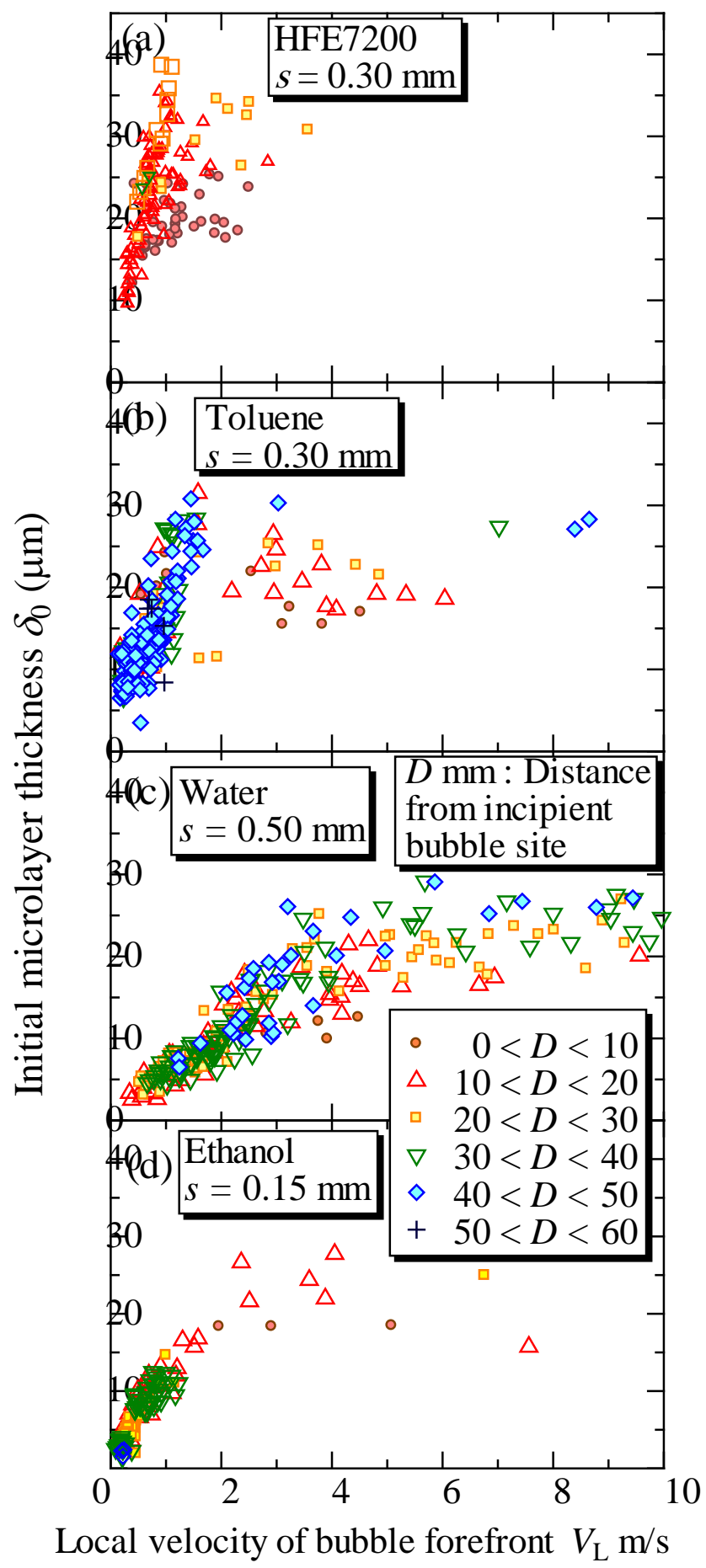

Fig. 5 


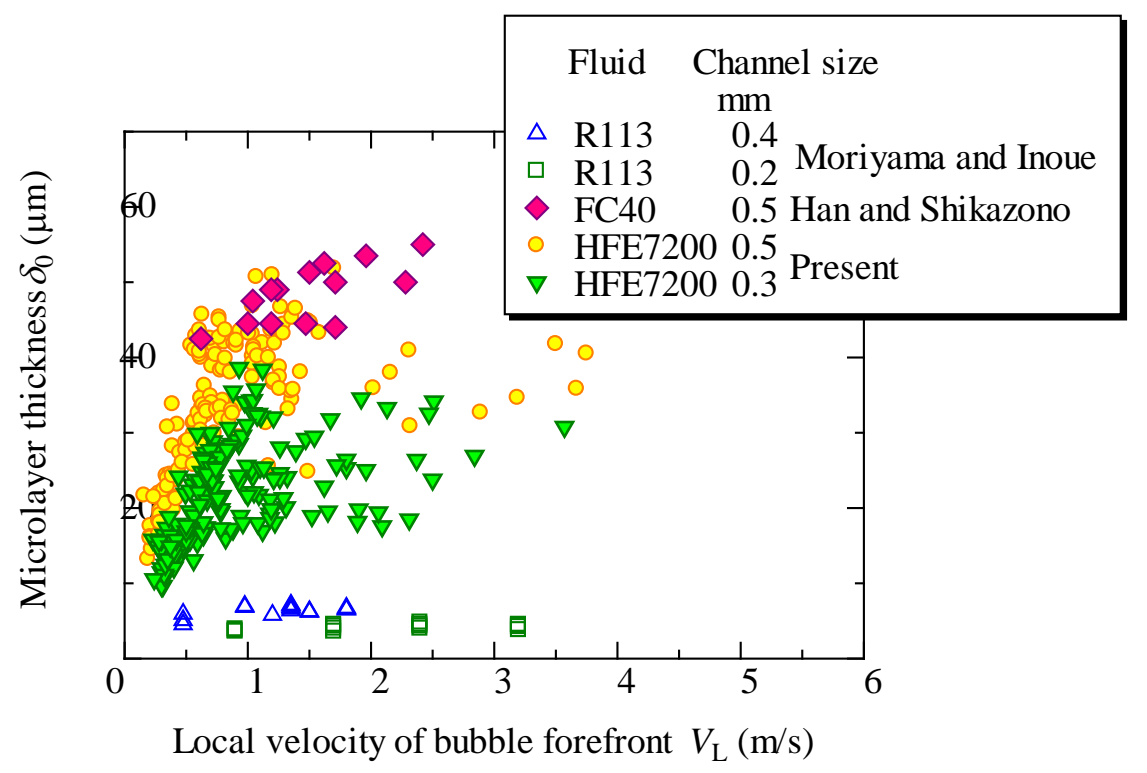

Fig. 6

Table 2

\begin{tabular}{lccc}
\hline & $\rho\left(\mathrm{kg} / \mathrm{m}^{3}\right)$ & $\mu(\mu \mathrm{Pa} \cdot \mathrm{s})$ & $\sigma(\mathrm{mN} / \mathrm{m})$ \\
\hline HFE7200 & 1307 & 330.0 & 9.4 \\
R113 & 1499 & 515.7 & 14.8 \\
FC40 & 1849 & 326.7 & 16.0 \\
\hline
\end{tabular}




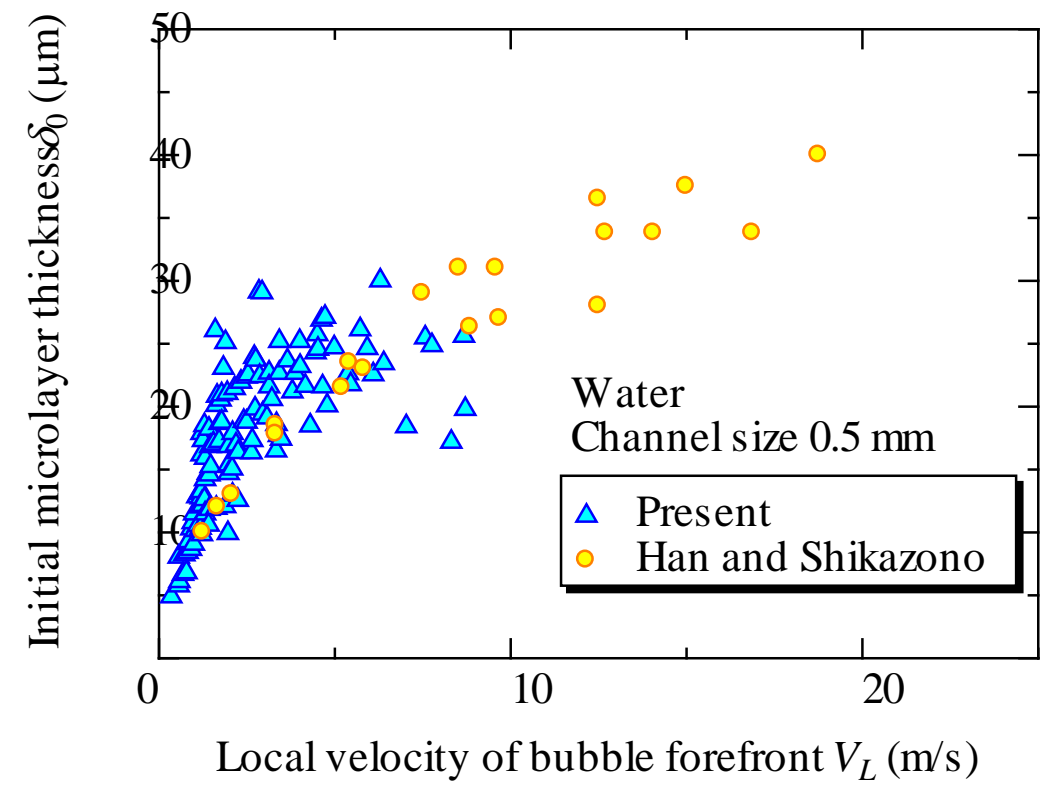

Fig. 7 


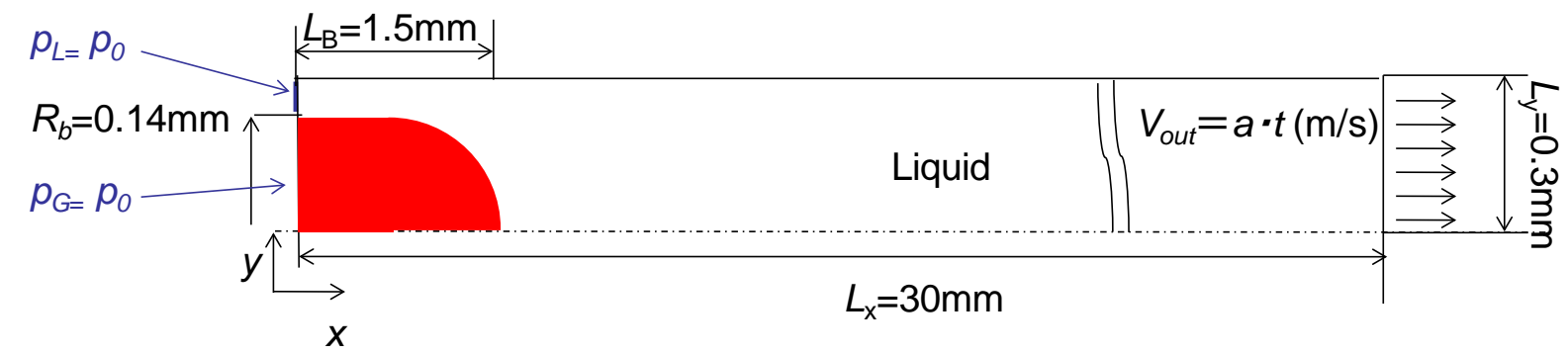

Fig. 8 


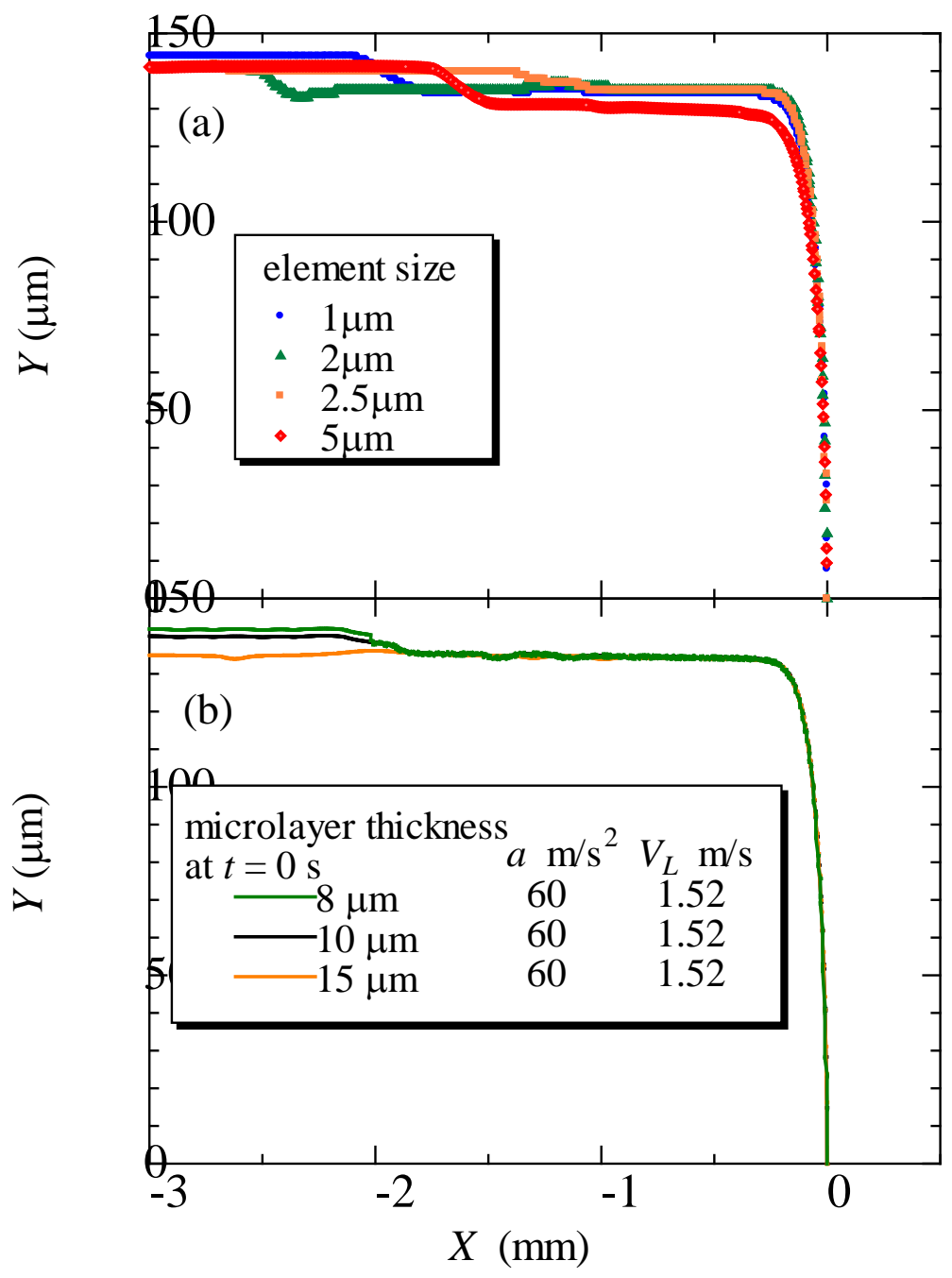

Fig. 9 

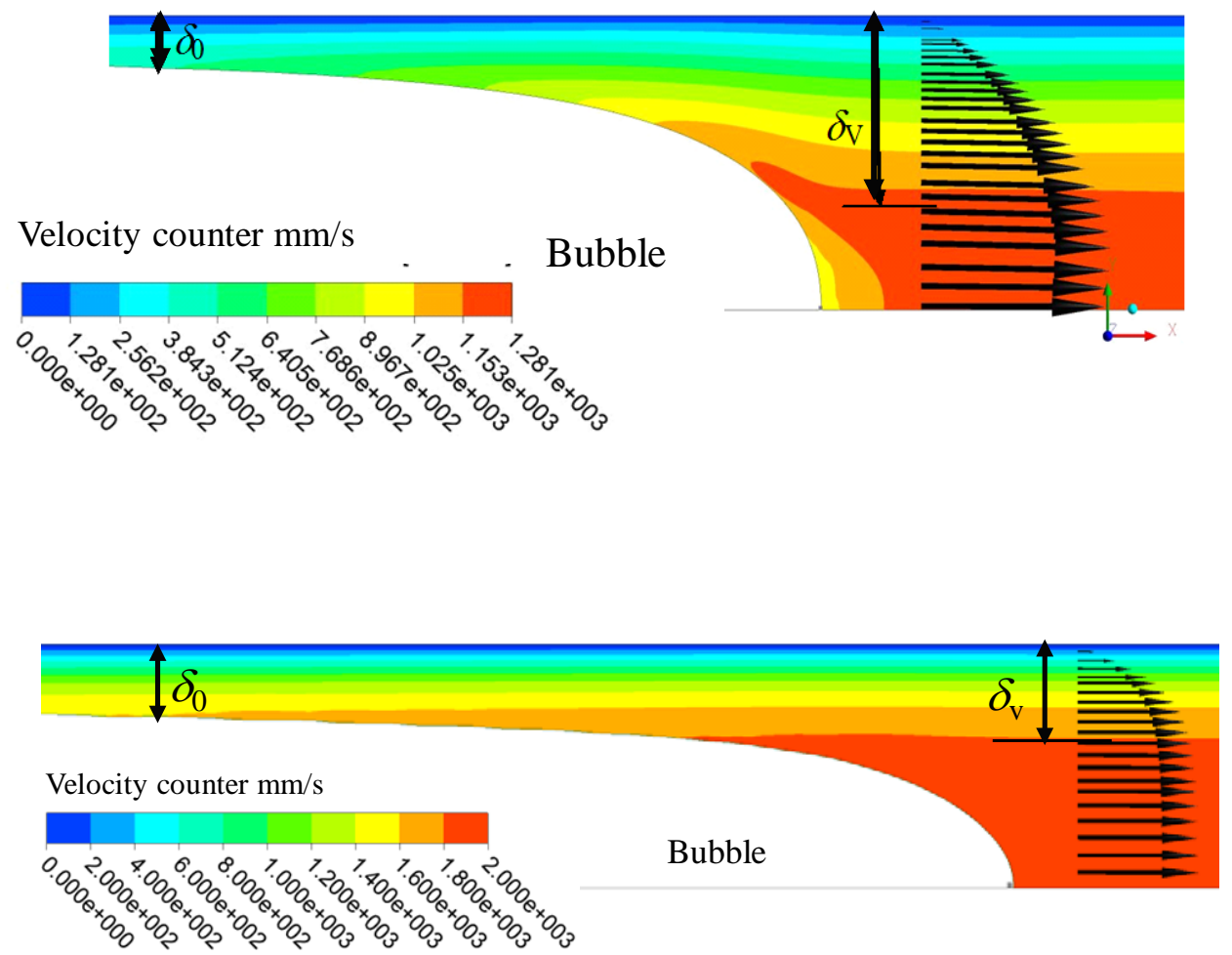

Fig. 10 


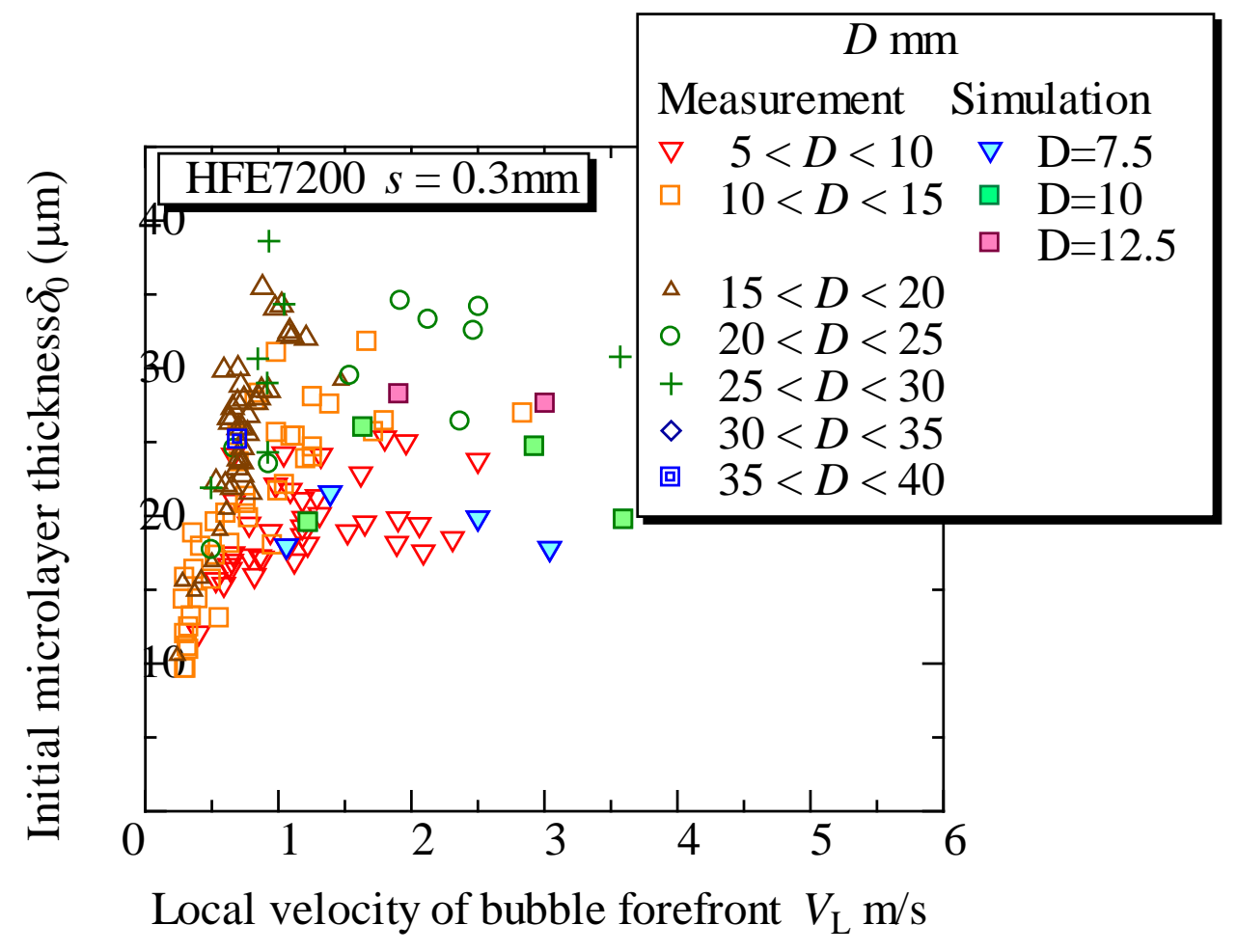

Fig. 11 


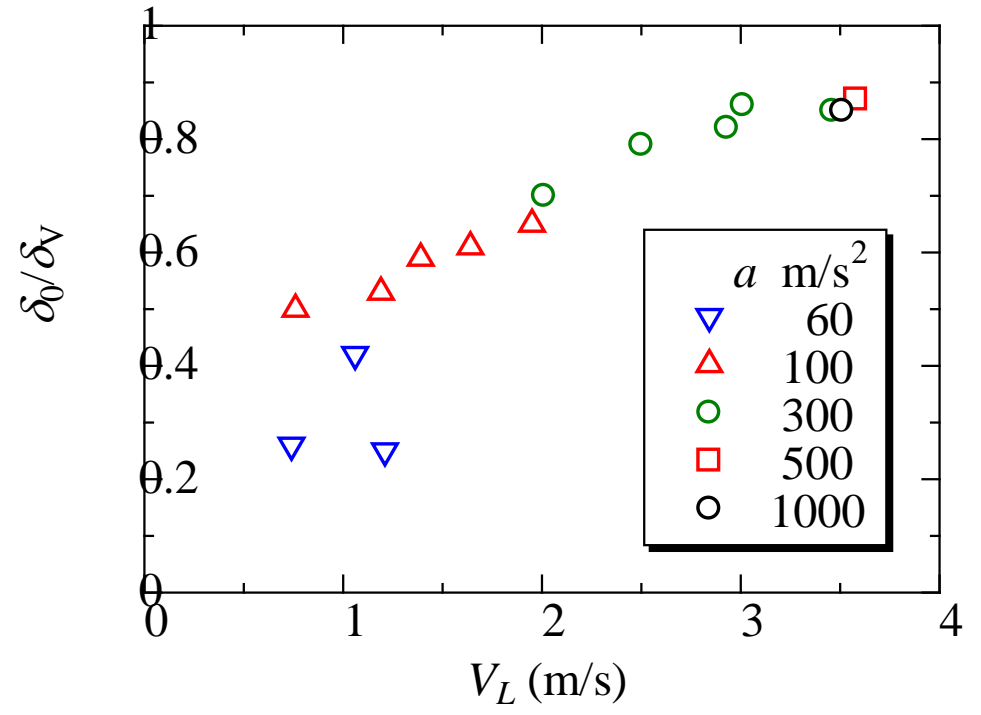

Fig. 12 


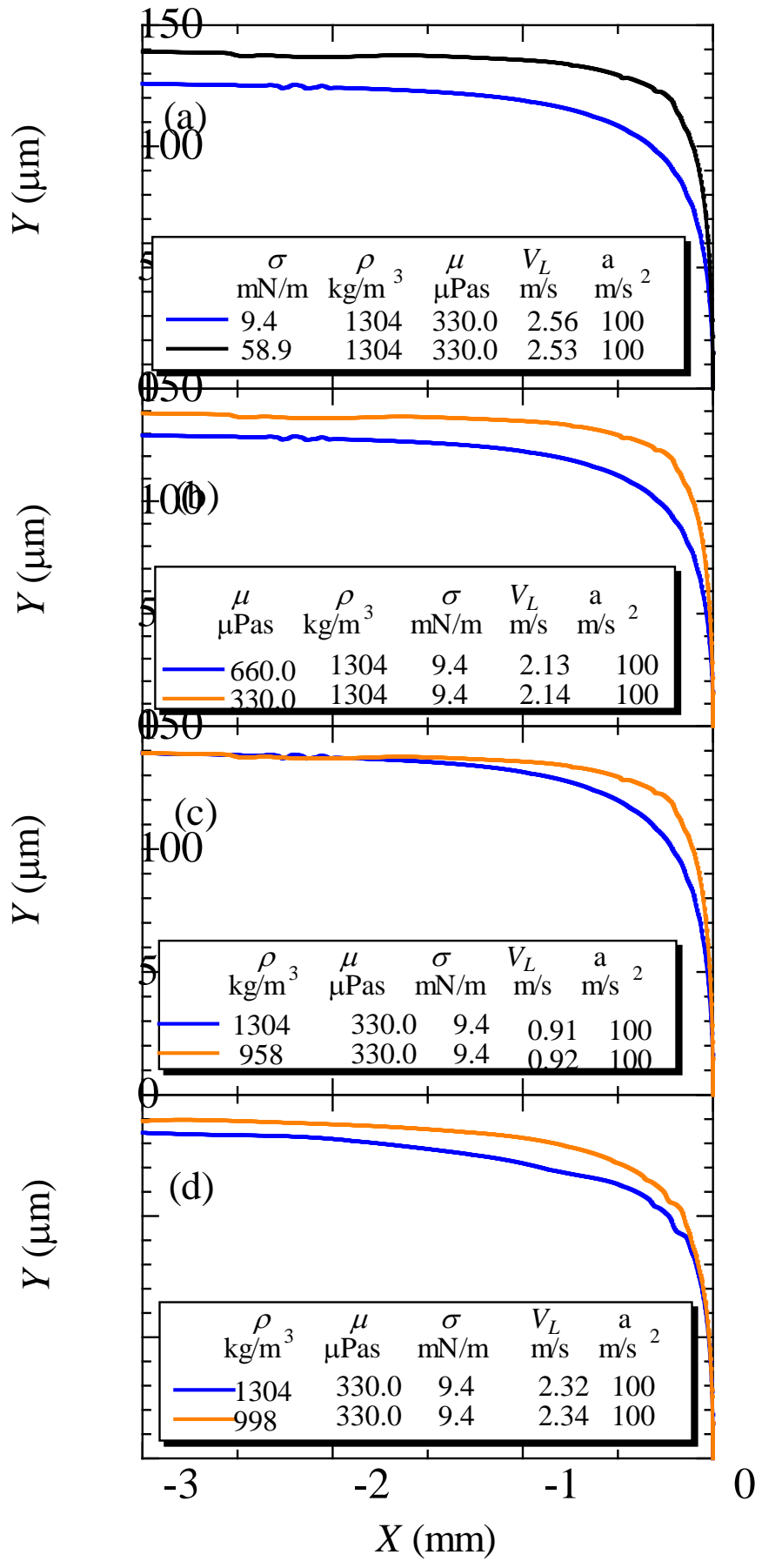

Fig. 13 


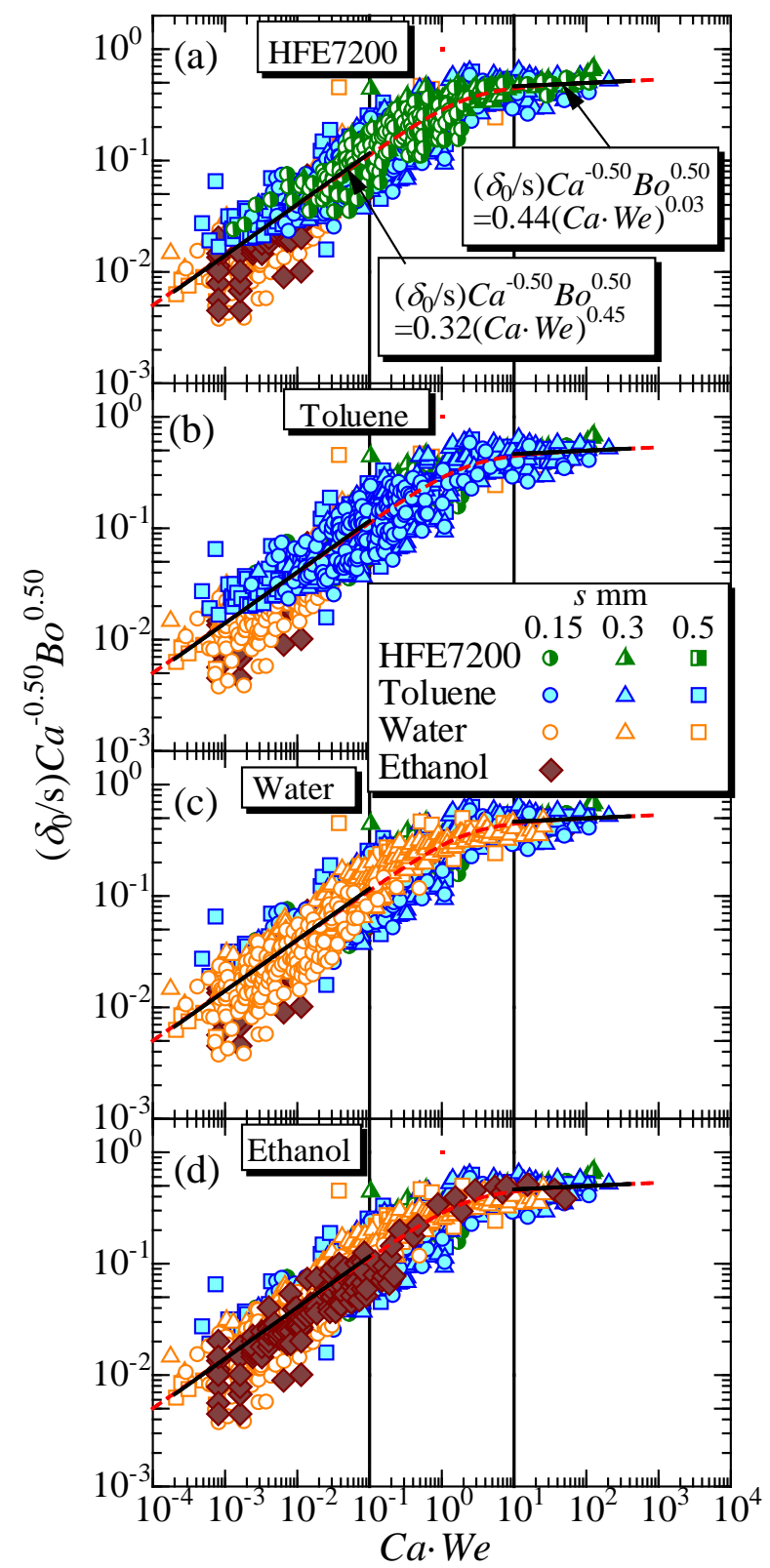

Fig. 14 


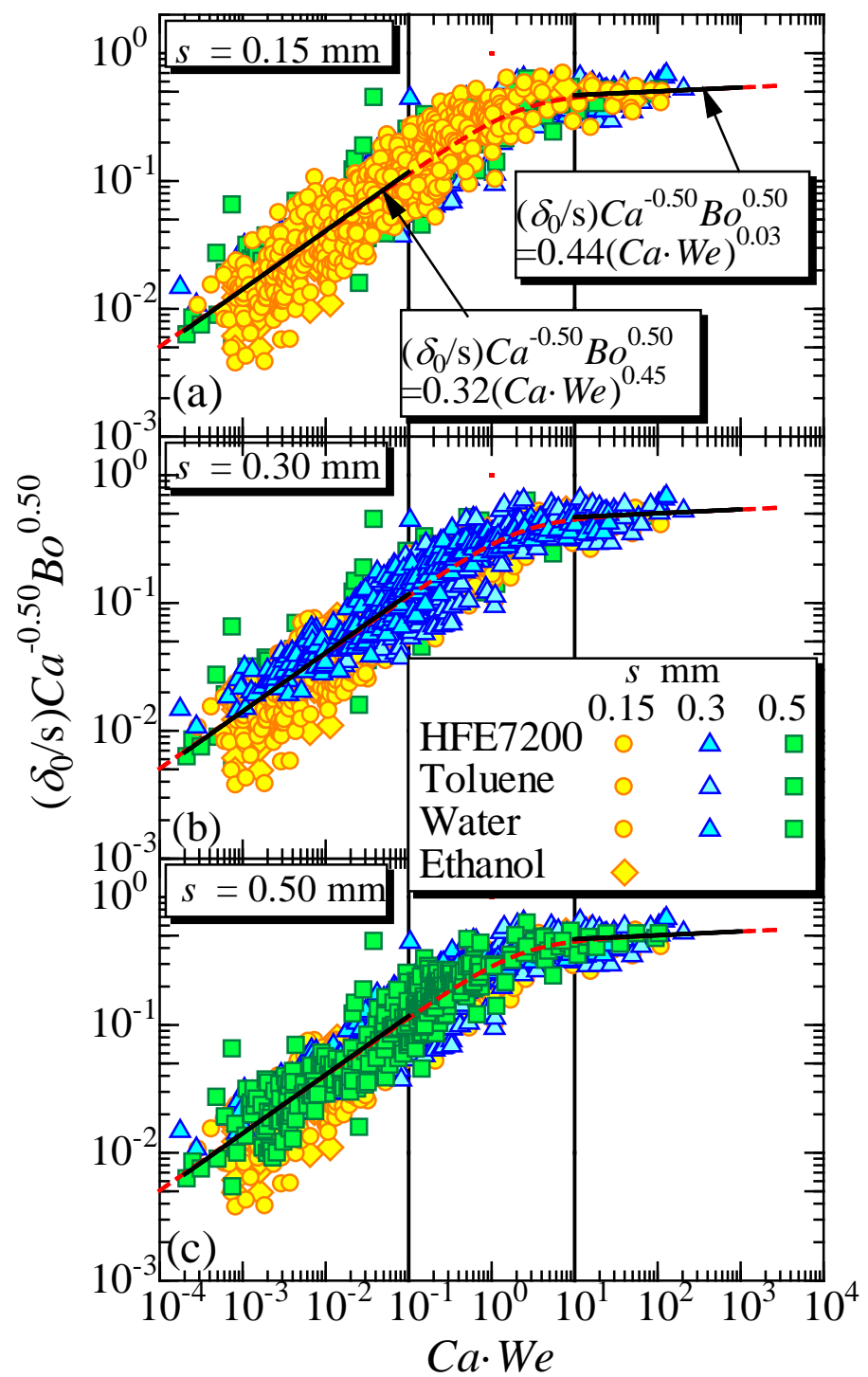

Fig. 15 


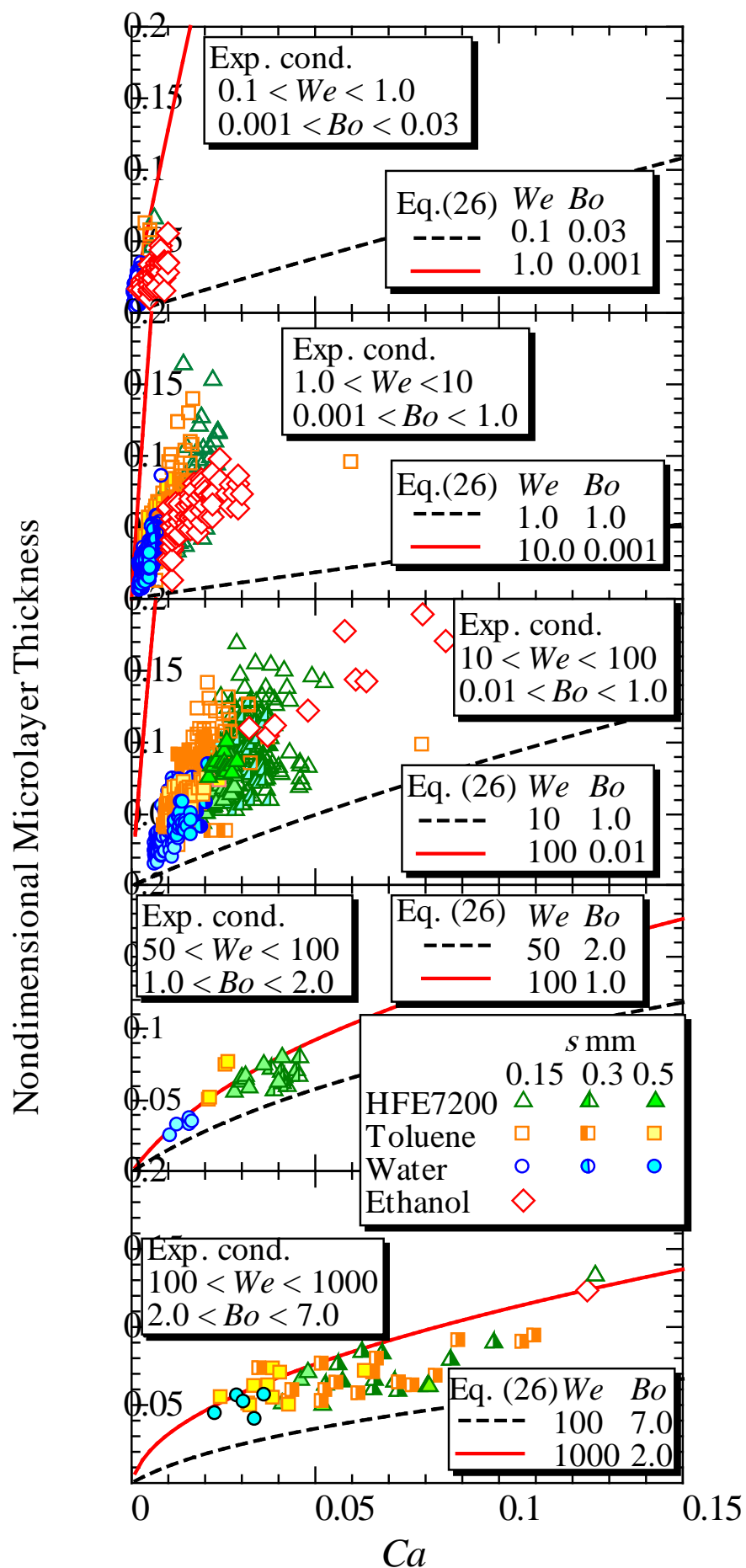

Fig. 16 